\author{
What strategies? For which territories? Communication \\ for prevention and promotion of health in the Buenos Aires \\ suburbs
}

Quais estratégias? Para quais territórios? Comunicação para

a prevenção e promoção da saúde nos subúrbios de Buenos

Aires

González Gartland, Georgina

Georgina González Gartland

ggartlan@campus.ungs.edu.ar

Universidad Nacional de General Sarmiento,

Argentina

REVCOM. Revista científica de la red de carreras de Comunicación Social

Universidad Nacional de La Plata, Argentina

ISSN: 2451-7836

Periodicidad: Bianual

núm. 12, e053, 2021

redcom.revcom@gmail.com

Recepción: 06 Julio 2021

Aprobación: 14 Julio 2021

URL: http://portal.amelica.org/ameli/

jatsRepo/203/2032361005/index.html

DOI: https://doi.org/10.24215/24517836e053

\section{(1) (0)}

Esta obra está bajo una Licencia Creative Commons AtribuciónNoComercial-CompartirIgual 4.0 Internacional.
Resumen: El objetivo de este artículo se centra en problematizar la intervención en comunicación en el territorio entendiendo que allí se entrecruzan relaciones económicas, sociales, culturales, políticas y comunicativas que operan sobre las representaciones y las acciones de las comunidades en torno a la salud, la enfermedad y la atención. Partimos de la comunicación como componente estratégico que pone en la agenda pública los temas y problemas de salud. Entendemos que en los procesos comunicacionales de salud-enfermedad-atención se ponen en juego herramientas e instrumentos diversos que abarcan desde los productos comunicativos que también son coproducidos desde los territorios. Por ello, creemos necesario visibilizar los claroscuros que presentan los territorios a la hora de diseñar e implementar estrategias de comunicación para la salud sin perder de vista los rasgos de los entornos tecnoculturales (Cabello, 2008) que suman complejidad al análisis.

Palabras clave: Estrategias de comunicación, territorios, salud.

Resumo: O objetivo deste artigo está focado em problematizar a intervenção comunicacional no território, entendendo que existem relações econômicas, sociais, culturais, políticas e comunicativas interseccionais que operam nas representações e ações das comunidades em torno da saúde, da doença e da atenção. Partimos da comunicação como um componente estratégico que coloca as questões e problemas de saúde na agenda pública. Entendemos que nos processos comunicacionais de saúde-doença-cuidado são colocados em jogo diversos instrumentos e instrumentos que vão desde produtos comunicativos, mas também são coproduzidos a partir dos territórios. Portanto, acreditamos ser necessário tornar visível o claro-escuro apresentado pelos territórios na concepção e implementação de estratégias de comunicação para a saúde, sem perder de vista as características dos ambientes tecnoculturais (Cabello, 2008) que acrescentam complexidade à análise. 
Palavras-chave: Estratégias de comunicação, territórios, saúde. Abstract: The objective of this article is focused on problematizing the communication intervention in the territory, understanding that there are intersecting economic, social, cultural, political and communicative relationships that operate on the representations and actions of the communities around health, disease and attention. We start from communication as a strategic component that puts health issues and problems on the public agenda. We understand that in the communicational processes of health-disease-care diverse tools and instruments are put into play that range from communication products but are also co-produced from the territories. Therefore, we believe it is necessary to make visible the chiaroscuro presented by the territories when designing and implementing communication strategies for health without losing sight of the features of technocultural environments (Cabello, 2008) that add complexity to the analysis.

Keywords: Communication strategies, territory, health.

\section{Estrategias de COMUNiCACión EN SALUd: PREguntas PARA PRODUCIR CON Y PARA EL TERRITORIO}

En América Latina, los desarrollos teóricos del campo de la comunicación y la salud, desde mediados del siglo XX, identificaron, caracterizaron y definieron su rol e incidencia en diferentes ámbitos de la vida social. Los avances más significativos se produjeron en el espacio de la comunicación pública con los estudios de las campañas electorales, la comunicación gubernamental ${ }^{1}$ o de políticas públicas. De igual modo se formalizaron investigaciones e intervenciones en el ámbito educativo, productivo y de salud en el marco del modelo de Difusión de Innovaciones ${ }^{2}$ como también en relación con los medios de comunicación y el discurso publicitario, entre otros.

En esta heterogeneidad y complejidad de perspectivas y abordajes, la comunicación y la salud conforman un espacio interdisciplinario en construcción y expansión, según señala Rodríguez Zoya (2017). La producción académica y profesional se ha dedicado principalmente a la salud en los medios, la comunicación institucional médica, la comunicación de políticas públicas, la comunicación de riesgos, campañas públicas. Sin embargo, más allá de la multiplicidad de enfoques y las diversas experiencias, se ha problematizado en menor medida la intervención en comunicación en salud en los territorios. De allí, que nos interesa aportar algunas observaciones contextuales sobre esta área de vacancia y otras que pueden colaborar con la visibilización de algunas pocas certezas y muchas más preguntas que requieren seguir estudiando y compartiendo saberes y prácticas de dicha relación.

Nuestra entrada al campo de estudios de comunicación y salud se propone identificar algunos de los desafíos para pensar estrategias de comunicación en salud escalando el concepto de territorio. Pero ¿̇cuándo y dónde pensamos estos desafíos? Claramente no podemos hacerlo sino en contexto, en el actual, en el que nos urge y nos interpela. No pueden ser considerados fuera de estos tiempos de crisis pero fundamentalmente tampoco por fuera de los territorios.

La necesidad de comprender el territorio como el espacio social e históricamente construido (Chiara, 2018; Becerra \& Borcoski, 2020) nos permite reflexionar sobre las lógicas que encarnan las estrategias de comunicación pública para la prevención y la promoción de la salud de las poblaciones del conurbano bonaerense. Estas estrategias se insertan en ambientes más despojados de imágenes y de dispositivos 
tecnológicos, donde persiste la presencia de medios de comunicación de masas ( $\mathrm{TV}$, radio o libros) y en menor medida medios informáticos. Este paisaje tecnocultural discontinuo ligado al acceso desigual a las tecnologías digitales resulta preciso para analizar la comunicación en el conurbano bonaerense. Aclaramos aquí que no nos proponemos realizar ningún tipo de generalización sobre toda la región metropolitana de Buenos Aires sino que nos preocupa trazar algunas claves de lectura para mirar la relación entre comunicación, salud y territorio.

En este marco, nos centraremos en problematizar la intervención en comunicación en el territorio entendiendo que allí se entrecruzan relaciones económicas, sociales, culturales, políticas y comunicativas que operan sobre las representaciones y las acciones de las comunidades en torno a la salud, la enfermedad y la atención. ${ }^{3}$

Partimos de la comunicación como componente estratégico que pone en la agenda pública los temas y problemas de salud. Entendemos que en los procesos comunicacionales de salud-enfermedad-atención se ponen en juego estrategias diversas que abarcan desde los productos, los procesos y las prácticas comunicativas que también son coproducidas desde y en los territorios. Por ello, creemos necesario reconocer los contrastes y tensiones que presentan los territorios a la hora de diseñar e implementar estrategias de comunicación para la salud sin perder de vista los rasgos de los entornos tecnoculturales (Cabello, 2008) que suman complejidad al análisis.

De hecho, como mencionamos más arriba, tenemos más dudas que certezas pero nos preceden debates, experiencias e investigaciones que nos alientan a abrir otros campos de preguntas que oficien de impulso para seguir produciendo conocimiento en comunicación y salud para y con las poblaciones de nuestro conurbano bonaerense.

\section{EL TERRITORIO Y EL ENTORNO TECNOCULTURAL PARA PENSAR LA COMUNICACIÓN PARA LA SALUD}

Si nos detenemos a reflexionar sobre la formulación de políticas de salud ${ }^{4}$, la dimensión comunicativa se presenta como un eje importante en la disposición de procesos de intervención, implementación y sostenibilidad de esas políticas. Entre los diferentes componentes para ejecutar una política de salud nos enfocamos en la necesidad de su comunicabilidad: los contenidos y el modo que adquieren sus estrategias deben ser puestas a disposición de la población destinataria de forma clara y precisa. Es en este punto donde creemos que no debemos dejar de precisar el modo en que la complejidad del territorio y sus demandas interpela la gestión de las políticas de salud.

En principio, retomamos la postura de Tobar (2010) que comprende el abordaje territorial en salud como un proceso integral que busca satisfacer las demandas de la población en el territorio. De allí que la comunicación tiene un rol relevante para articular la gestión de las políticas de salud en diálogo con el territorio.

Ahora bien, como decíamos más arriba, nos importa problematizar la noción de territorio para analizar la comunicación de las políticas de salud expresadas en estrategias que -entre sus elementos- comprenden desde la comunicación masiva, la interpersonal, la educación, hasta las formas tradicionales “(...) y específicas de una cultura, tales como la narración de historias, guiñoles y canciones” (OMS, 1998, p. 19).

Los debates en torno al territorio provienen de diferentes campos disciplinares tales como la geografía, la sociología, los estudios urbanos, la economía política, entre otros, cuyos estudios coinciden en la polisemia del concepto. ${ }^{5}$ La idea de territorio se suele asociar a los límites físicos también como espacio productivo, simbólico, de relaciones sociales o de poder o en tanto, construcción de subjetividades. Wulff (2014) retoma el relevamiento que realiza Haesbaert (2011) sobre esta multiplicidad de abordajes y registra 
cuatro perspectivas que consideran el territorio: la naturalista, la jurídico-política, la simbólico-cultural y la económica.

Sin embargo, la complejidad de los procesos sociales, políticos, económicos, culturales que involucra requiere de análisis contextualizados teniendo en cuenta las relaciones instituidas e instituyentes que se entraman. En esa dirección, Becerra y Borcoski (2020) -desde sus estudios sobre municipalismo- nos invitan a "territorializar el pensamiento" de modo tal que sostienen que:

El territorio adquiere dos formas: primera, la de una superposición de relaciones esenciales, entre los problemas, entre los actores, entre la humanidad y la biósfera, un espacio prioritario de valorización de los bienes que se multiplican al compartirse; luego el lugar mismo en donde se organizan las relaciones entre los niveles de gobernanza (p. 176).

De este modo, la categoría de territorio adquiere relevancia para comprender las relaciones, las prácticas, la producción de sentido en las esferas de lo social, lo cultural, lo económico, lo político, etcétera.

No obstante, aquí nos interesa pensar las políticas de salud en el conurbano bonaerense ${ }^{6}$ que tensionan el concepto de territorio ensamblado a las prácticas comunicativas siempre en diálogo con la trama sociocultural en la que se producen. $\mathrm{Al}$ respecto y para poner en contexto el análisis recuperamos la noción de entorno tecnocultural que desarrolla Cabello (2008):

La oferta y disposición de un conjunto cada vez más variado de dispositivos tecnológicos -en este caso, en general, digitalescontribuye con la configuración de un tipo particular de escenario en el cual se desarrollan los intercambios sociales, que se diferencia sustantivamente de otros que predominaron en otros momentos históricos (p. 2).

La autora señala que uno de los rasgos de este entorno es su carácter de inestabilidad dado por la marca de la obsolescencia propia de los dispositivos tecnológicos (nuevos productos en el mercado que varían en sus aspectos funcionales o estéticos, nuevos usos generados por los sujetos que se vuelven más competentes con su propia práctica); y que, por lo tanto, resulta cambiante convirtiéndose en un "telón de fondo". Un escenario heterogéneo que se torna "medio" y, que "(...) asume un carácter envolvente que se materializa en cada uno de los espacios y momentos en que las personas interactúan entre sí, realizan sus actividades y, además, se relacionan interactivamente con las máquinas" (Cabello, 2008, p. 2). Y advierte que no hay que perder de vista que este ambiente se apoya en la lógica del mercado, que a través de la producción y distribución de tecnologías a escala mundial, produce una gran penetración de los dispositivos a la vez que profundiza las desigualdades en el acceso social.

Cabello (2008) reconoce que ese nuevo tipo de escenario adquiere características distintas según se trate de centros urbanos o de lo que llama "paisajes periurbanos" como por ejemplo, la Ciudad de Buenos Aires en relación con el Área Metropolitana (conurbano bonaerense). En el caso de los paisajes periurbanos, la investigadora sostiene que el entorno tecnocultural se manifiesta como "discontinuo" debido a que se encuentra más despojado de imágenes y de dispositivos tecnológicos y donde persiste la presencia de medios de comunicación tradicionales (TV, radio, prensa) y, en menor grado, tecnologías digitales. Este paisaje tecnocultural discontinuo ligado al acceso desigual a las tecnologías digitales, a diferentes grados de apropiación de los dispositivos, reviste algunos de los atributos que distinguen el entorno tecnocultural en las zonas periféricas.

Estas conceptualizaciones sobre los rasgos del territorio y de la cultura actual nos permite situar el abordaje de la comunicación de las políticas de salud en el contexto socioeconómico del conurbano bonaerense.

Así es que seguimos la línea de trabajo de Chiara (2020) que entiende al territorio “(...) como variable importante al momento de diseñar estrategias sanitarias" (p. 28) Y continúa planteando que en el diseño de las políticas el aporte de la salud comunitaria presenta un "(...) antecedente (...) valioso que se esfuerza por recuperar las condiciones del territorio en la forma de concebir y llevar adelante las acciones en salud (...)" (p. 29).

Desde este enfoque, el territorio es un escenario donde se construyen y reconstruyen las relaciones sociales, históricas, políticas, culturales, económicas y ambientales donde la tríada salud-enfermedad-atención expresa 
tensiones y conflictos. Esto muestra la necesidad de que las políticas sanitarias se apropien de la categoría de territorio para comprender e intervenir con acciones orientadas a la prevención y a la promoción de la salud. A la vez que, para diseñar las estrategias de comunicación de esas políticas, resulta significativo comprender que en ese escenario se manifiesta un entorno tecnocultural caracterizado principalmente por el acceso desigual y una debilitada apropiación de los medios de comunicación y las tecnologías digitales.

\section{INTERVENCIÓN DE LA COMUNICACIÓN EN SALUD EN EL TERRITORIO: EL CONURBANO EN CONTEXTO}

El campo de la intervención en comunicación requiere reflexionar sobre las modalidades que adquieren las concepciones vigentes en la sociedad, las perspectivas comunicacionales pero principalmente acerca de las características a partir de las cuales los actores se posicionan y participan de la dinámica social. Por ende, los modos de intervención comunicacional de las políticas y acciones de salud se vinculan no solo con el papel que le asigna el Estado en sus distintos niveles de gestión, la definición y ejecución por parte de los decisores desde sus posicionamientos sino también, y particularmente, en relación con la concepción de desarrollo vigente. De esta manera, la perspectiva de desarrollo predominante involucra decisiones políticas y estratégicas que caracterizan la modalidad propia de comunicación de políticas, programas y proyectos de salud en el territorio.

En esta línea, Cimadevilla se propone repensar la dimensión comunicativa en los procesos de desarrollo, particularmente centra su objeto de análisis en la intervención como modo de difusión. Plantea que la comunicación es la cara visible de toda intervención y que es ahí donde hay que poner el centro de atención a la hora de abordar las estrategias comunicativas de políticas, programas o proyectos.

La idea de la intervención (...) puede concebirse a la luz de una acción que involucra una posición, sin que necesariamente sea coercitiva. El papel de la comunicación, justamente, podría ser el de proponer que la legitimación necesaria surja del protagonismo de los actores, más que de la persuasión de los autores (Cimadevilla, 2004, p. 137).

Estas presunciones permiten entender la intervención en términos de acciones llevadas a cabo en el escenario de las prácticas, acompañando los procesos históricos. Una intervención que lleva a posicionarse en las estrategias, estrategias que son definidas y ejecutadas por todos los actores involucrados en la resolución de los problemas en cuestión. Esta noción supone que todos aquellos que participan ponen en juego sus propias estrategias comunicativas. Es decir, es en la relación de los actores en la que intervienen las estrategias de comunicación donde centramos nuestra mirada al momento de analizar la presencia y la/s incidencia/s de las políticas, programas y/o acciones sanitarias en el territorio.

Por lo tanto, situar la reflexión sobre la intervención en comunicación en salud en el conurbano bonaerense requiere enfocar las dinámicas y tensiones en el territorio que se visibilizan en el acceso desigual a servicios básicos (agua, vivienda, energía eléctrica, gas natural, red cloacal, etc.) así como también, en un entorno tecnocultural discontinuo. Estos condicionamientos materiales y simbólicos combinados presentan ciertas características que son objeto de indagación en el ámbito académico tanto como en el de las políticas y/o acciones de salud. En nuestro caso, repasaremos aquellas que se ajustan al diseño estratégico de la comunicación en parte del conurbano bonaerense a cuenta de contribuir con la discusión sobre la co construcción de las políticas de salud en territorio.

Uno de los puntos de partida que proponemos es tomar distancia de las perspectivas instrumentales tanto en relación con la comunicación - en términos de difusión de la información - como con la categoría de territorio -como espacio físico delimitado.

El rasgo principal del conurbano bonaerense, según sostiene Rofman (2014) no se basa solamente en el tamaño del territorio y el conjunto de su población sino además en “(...) la escala de sus principales actividades 
y de los flujos de población y de intercambio de bienes y servicios que la recorren cotidianamente y que atraviesan -y en cierta medida, desconocen- los límites jurisdiccionales entre municipios (p. 2).

A esta vasta estructura político-institucional se suma la expansión de la mancha urbana, caracterizada por el aumento de población con necesidades básicas insatisfechas a medida que se distancia de la Ciudad Autónoma de Buenos Aires, que tiene rasgos "(...) de descoordinación y escasa planificación de ese crecimiento" (Soldano, 2014, p. 12) Estos datos se cruzan indefectiblemente con una fuerte desigualdad habitacional ${ }^{7}$ que se combina con un déficit de infraestructura básica ${ }^{8}$ en medio de una seria degradación ambiental, producto de una presencia discontinua y clientelar de planificación estatal.

La convivencia de los factores socioeconómicos, demográficos y espaciales inscribe la lógica del barrio entre las poblaciones de la región donde las respuestas a los problemas sociales, habitacionales o ambientales se producen y se afirman en las redes de proximidad. ${ }^{9}$ Es en estas redes de proximidad donde se construyen distintas modalidades de comunicación entre los diferentes actores del territorio (los y las vecinos/as, las organizaciones sociales, los niveles de gestión gubernamental, etc.) que van desde lo interpersonal, lo masivo hasta las mediadas por tecnologías. Siempre teniendo en cuenta que estos vínculos se manifiestan en un paisaje periurbano con un marcado acceso diferencial de dichas tecnologías digitales, desiguales grados de apropiación de los dispositivos que ratifica la marca de la periferia (Cabello, 2008, p. 4). Estos rasgos nos habilitan a poner el foco en las estrategias de comunicación para la prevención y promoción de la salud.

Como decíamos al inicio de este trabajo no podemos dejar de reconocer estas prácticas y procesos en el territorio sino es en contexto. A tal efecto, pretendemos recuperar las principales condiciones socio sanitarias pre pandemia covid-19 en relación con el primer tramo de la misma durante el 2020. Para ello, revisaremos brevemente dos experiencias que dan cuenta de la relevancia que encarna el territorio como elemento catalizador que, desde nuestro punto de vista, necesariamente opera en el diseño, definición, redefinición, la implementación de las estrategias comunicativas en salud.

Al borde la pandemia en nuestro país -fines de 2019- se comenzó a desarrollar una iniciativa de comunicación en el primer nivel de atención de salud en un partido del noroeste del conurbano bonaerense. ${ }^{10}$ La propuesta fue llevar a cabo una estrategia de intervención sostenida en el asesoramiento técnico y la capacitación en comunicación y salud para trabajadoras sociales (TS) que se desempeñaban en unos 20 centros de atención primaria de la salud (CAPS). Lo valioso de este proyecto fue que se originó a partir de una demanda explícita de las TS que reconocían no contar con competencias y habilidades comunicativas en clave intercultural para abordar las diferentes situaciones en el plano de la atención de los usuarios de los CAPS.

El primer nivel de atención es la puerta de entrada al sistema de salud cuya principal función es ofrecer los cuidados de salud tan cerca como sea posible al lugar donde las personas viven y trabajan. Constituye el primer eslabón de la atención que involucra a la comunidad que debe formar parte activa del proceso en acciones preventivas y de autocuidado. Uno de los principales inconvenientes que relataban las profesionales en los CAPS fue la coexistencia de situaciones socioeconómicas y ambientales críticas experimentadas por los y las usuario/as del sistema sanitario y las urgencias o emergencias propias de salud. En el transcurso de la atención percibían que quedaba, por lo menos, en forma precaria la acción comunicativa que permitiría un acercamiento a las familias más efectivo por parte de las TS. Esta evaluación entre las TS y el equipo del proyecto permitió construir un espacio multidisciplinario de reflexión-acción para producir estrategias de comunicación adecuadas a las necesidades en el primer nivel atención de la salud.

Iniciado el 2020 y ya declarado el A.S.P.O. otra experiencia fue promovida por un conjunto de organizaciones sociales de otro partido del conurbano bonaerense en articulación con 3 universidades. ${ }^{11}$ En este caso, se planteó capacitar en los barrios y producir herramientas de comunicación para asistir y difundir medidas de cuidado a quienes estaban acompañando a las vecinas y los vecinos en los barrios. El avance de los contagios obligó a reorientar la estrategia a una modalidad virtual tanto para las actividades de capacitación ${ }^{12}$ como para las acciones de comunicación. Cuando se dispuso la fase del D.I.S.P.O. se decidió 
seguir acompañando a los y las referentes y a vecinos y vecinas de los barrios en el sostenimiento de las medidas de cuidado y reforzando la comunicación de las medidas de prevención de la salud en la progresiva apertura de actividades.

Los hallazgos y las decisiones tomadas sobre la comunicación en el marco de este proyecto fueron sostenidas a partir de identificar que las prácticas comunicativas de los y las vecinos y vecinas de los barrios se asentaban en las comunicaciones mediadas por tecnologías: redes sociales como el WhatsApp y Facebook. De allí que la producción de piezas de comunicación (videos, audios y piezas gráficas) tuvo en cuenta los lenguajes, las percepciones, los sentidos y las representaciones de los y las actores sociales de los barrios.

Por un lado, se produjeron materiales de apoyo a la capacitación que se materializaron en el diseño de fichas que adaptaron las orientaciones de los protocolos nacionales y provinciales que a la vez pusieron en circulación los y las participantes mediante redes sociales. ${ }^{13}$ Por otro lado, se elaboraron piezas en diferentes formatos con instrucciones para acceder a los cursos, con recomendaciones, con mensajes producidos por y para los vecinos y las vecinas de los barrios que se pusieron a disposición en un canal de You Tube.

Ambas propuestas de intervención, que se implementaron antes y durante la pandemia COVID 19, comparten ciertas características que tienen al territorio como variable clave para analizar y diseñar estrategias de comunicación en contextos de desigualdad socio económica y cultural. Tomando en cuenta que estas intervenciones se produjeron en entornos tecnoculturales con una deficiente conectividad, obsolescencia de dispositivos y usos limitados de las tecnologías digitales.

Entre los rasgos que tienen en común estos proyectos podemos señalar la identificación de las problemáticas sociosanitarias por parte de los actores del territorio, tanto en el caso de las TS, de los y las referentes de las organizaciones como de los vecinos y las vecinas de los barrios. Estas demandas no se construyeron sin tensiones y conflictos propios de las condiciones materiales y simbólicas en las que se posicionaban.

Las TS, ubicadas en el primer nivel de atención, resignificaron sus propias prácticas dentro del sistema de salud entendiendo que la atención a las poblaciones usuarias requería de aportes de estrategias de comunicación que asociaban a las barreras a acceso a los centros de salud tales como: limitaciones en las prestaciones ofrecidas y en la cantidad de profesionales asignados, recursos y equipamientos limitados así como también horarios y días de atención reducidos.

Por otra parte, los y las referentes de las organizaciones sociales como los y las vecinas demandaron saberes y conocimientos prácticos para asistir, acompañar, difundir las medidas de cuidado y sobre los grupos de riesgo de covid-19. Pero la emergencia sanitaria aparecía como un aspecto crítico más superpuesto al deterioro de las condiciones de vida, productivas y laborales en los barrios. Las necesidades expresadas por los y las integrantes de las organizaciones estuvieron orientadas a estrategias comunitarias que les permitiera desarrollar algún tipo de mediación entre las acciones estatales, tales como Programa "El Barrio Cuida al Barrio" o el DETeCTAr ${ }^{14}$ y los territorios que consideraran las condiciones sociales y culturales de los mismos.

\section{LA COMUNICACIÓN DE PROXIMIDAD EN EL CONURBANO BONAERENSE}

Al inicio de este artículo nos preguntamos ¿cuáles son las estrategias de comunicación, para qué territorios? A lo largo de estas páginas tratamos de poner en tensión la relación comunicación, salud y territorio con el objeto de aportar elementos de análisis no solamente en tiempos de SARS-CoV-2 sino con la expectativa de que abran preguntas y líneas de acciones estratégicas pospandemia.

La segregación territorial con una escasa densidad de infraestructura de telecomunicaciones, heterogénea disponibilidad de dispositivos técnicos y reducidos usos de tecnologías digitales proyecta un acceso desigual a la provisión de servicios tecnológicos y culturales tanto por parte del mercado como del lado de la acción del Estado. Esa fragmentación sociodemográfica del territorio (grandes sectores de la población de bajos ingresos con deficientes servicios básicos, bajo nivel de instrucción, alta desocupación que coexisten junto 
a un reducido sector de altos ingresos) se entrama en el marco de un escenario en el que la distribución y penetración de tecnologías y la oferta cultural responde a las lógicas de mercado.

Según las experiencias presentadas es necesario considerar seriamente estos condicionamientos para comprender que las estrategias de comunicación en el territorio no se consuman en espacios sociales y culturales homogéneos que comparten sentidos y representaciones en relación con la prevención y la promoción de la salud. Por el contrario, las desigualdades, rupturas y discontinuidades de ese paisaje tecnocultural operan en la intervención en comunicación.

De este modo, entendemos que la participación de las organizaciones, los movimientos, los activistas que habitan el territorio muestra algunos de los aprendizajes para co construir estrategias comunicativas de proximidad sostenidas en los vínculos comunitarios que sean susceptibles de ser apropiadas por los actores involucrados. O sea, creemos sustantivo que esos actores intervengan activamente para fortalecer la capacidad de cuidado, para promover información confiable y conocimiento sobre el cuidado de la salud y que se transformen en emisores y productores de sentidos en sus comunidades de referencia. Es ni más ni menos que comunicación de proximidad que se expresa en la escucha, en compartir lo que se pone en común en la vida cotidiana del territorio que permita avanzar hacia la promoción del cuidado colectivo basado en la solidaridad y la reciprocidad.

Claramente, quedan cuestiones inconclusas, dudas e incertidumbres propias de la complejidad de los procesos que nos propusimos abordar. No obstante, para continuar reflexionando sobre comunicación, salud y territorio tal vez sea el momento de abrir más preguntas para "territorializar el pensamiento" (Becerra \& Borcoski, 2020) y procurar construir en común en post de "territorializar para transformar” (Hernández y otros, 2021).

\section{ReFERENCIAS}

Becerra, M. J. y Borcoski, I. (2020). Las huellas del futuro. Apuntes municipales para una nueva constitución: economia politica y territorio. Santiago de Chile, Chile: Corporación Ciudad y Derechos.

Boisier, S. (2011). El territorio en la contemporaneidad (La recuperación de las políticas territoriales). Líder. Revista Labor Interdisciplinaria de Desarrollo Regional, (18), 9-24. https://www.revistaliderchile.com/index.php/lider chile/article/view/136

Cabello, R. (2008). Pliegues en la tecnocultura. Question, (17). Recuperado de https://perio.unlp.edu.ar/ojs/index.p $\mathrm{hp} /$ question/article/view/1137

Capel, H. (2016). Las ciencias sociales y el estudio del territorio. Revista Bibliográfica de Geografía y Ciencias Sociales, XXI(1149), 1-38. Recuperado de https://revistes.ub.edu/index.php/b3w/article/view/26319

Chiara, M. (2018). La salud gobernada. Política sanitaria en la Argentina 2001-2011. Los Polvorines, Argentina: Ediciones UNGS.

Chiara, M. (2020). Salud en territorios segregados Apuntes para pensar las políticas desde los aprendizajes que deja la pandemia. Debate Público. Reflexión de Trabajo Social, 10(20), 27-40. Recuperado de https://cutt.ly/XQ4v1YR

Cimadevilla, G. (2004). Dominios: crítica a la razón intervencionista, la comunicación y el desarrollo sustentable. Ciudad Autónoma de Buenos Aires: Prometeo.

Hernández, M. y otros (2021). La pandemia de la enfermedad por coronavirus (COVID-19) desde una perspectiva crítica histórico-territorial. En C. Tetelboin Henrion, D. Iturrieta Henríquez y C. I. Schor-Landman (Coords.), América Latina. Sociedad, politica y salud en tiempos de pandemia. Ciudad Autónoma de Buenos Aires, Argentina: CLACSO.

INDEC (2010). Censo Nacional de Población y Vivienda. Publicaciones del INDEC, Ciudad Autónoma de Buenos Aires. Disponible en http://www.indec.gov.ar. [Consulta: 5 de marzo de 2015]. 
Llanos-Hernández, L. (2010). El concepto del territorio y la investigación en las ciencias sociales. Revista Agricultura, Sociedad y Desarrollo, 7(3), 207-220. Recuperado de https://www.revista-asyd.mx/index.php/asyd/article/view $/ 1119$

Mazurek, H. (2009). Espacio y territorio. Instrumentos metodológicos de investigación social. La Paz, Bolivia: IRD Éditions.

OMS (1998). Glosario de promoción de la salud. Ginebra, Organización Mundial de la Salud.

Rodríguez Zoya, P. G (2017). "Notas para pensar la complejidad del campo de Comunicación y Salud". En Petracci, M.; Schwarz P. K.N.; Rodríguez Zoya, P. G. Comunicación y Salud. Las relaciones entre médicos y pacientes en la Modernidad Tardia -1a ed.- Ciudad Autónoma de Buenos Aires: Teseo.

Rofman, A., (2014). Territorio, Sociedad y Política en la Región Metropolitana de Buenos Aires. Boletín electrónico $\mathrm{N}^{\circ} 1$ del Observatorio del Conurbano Bonaerense. Recuperado de http://observatorioconurbano.ungs.edu.ar/ $? \mathrm{p}=912$

Soldano, D. (2014). El conurbano bonaerense como expansión, desigualdad y promesa. Revista de la Facultad de Ciencias Sociales, (86), 12-16. Recuperado de http://www.sociales.uba.ar/wp-content/uploads/S86-DOSSIER -SOLDANO.pdf

Tobar, F. (2010). Gestión territorial en salud. En M. Chiara y J. Moro (Comps.), Pensando la agenda de la politica. Capacidades, problemas y desafios (pp. 15-44). Los Polvorines, Argentina: Universidad Nacional de General Sarmiento.

Tobar, F. (2015). Módulo 1. Salud y cuestión social. En M. Chiara (Comp.), Gestión territorial integrada para el sector salud. Los Polvorines, Argentina: Universidad Nacional de General Sarmiento.

Wulff, P. (2014). Mapa y territorio. Politicas públicas, comunicación y territorio. Mimeo. Recuperado de https://www.academia.edu/10978126/Mapa_y_Territorio_Pol\%C3\%ADticas_p\%C3\%BAblicas_comuni caci\%C3\%B3n_y_territorio

\section{Notas}

1 Estos estudios se enmarcan en el paradigma Mass Communication Research, también conocido como Teoría de los Efectos Limitados que tratan de explicar el comportamiento de la audiencia frente a los medios de comunicación masiva en diferentes contextos: de crisis, de elecciones o de normalidad. Para profundizar el tema se puede consultar Wolf (1991): La investigación de la comunicación de masas. México DF, Paidós.

2 También denominado "Difusionismo", cuyo mayor exponente es el sociólogo norteamericano Everett M. Rogers. En la década del sesenta sienta las bases de la teoría en la obra "Difusión de Innovaciones" basada en un paradigma del cambio social a partir de la aplicación de técnicas de la comunicación en comunidades rurales tradicionales. Es decir, considera a la difusión de la innovación como motor de modernización de la sociedad.

3 Este artículo recoge parte de las discusiones y las experiencias en una universidad del conurbano bonaerense sobre intervención en comunicación y salud en el territorio desde fines de 2019 a la actualidad. Debido a que las propuestas han incluido la participación activa de referentes de la comunidad y de organizaciones sociales del área de referencia de dicha casa de estudios, hemos decidido no dar a conocer datos de los proyectos para evitar revelar la identidad y rol de los actores territoriales involucrados.

4 Cuando hablamos de políticas de salud nos referimos a aquellas políticas “(...) definidas como un esfuerzo sistemático para reducir los problemas de salud. Una política de salud implica la definición de la salud como un problema público en el cual el Estado asume un rol activo y explícito" (Tobar, 2015, pp. 12-13).

5 Se pueden consultar los trabajos de Becerra y Borcoski (2020), Capel (2016), Boisier (2011), Llanos-Hernández (2010), Mazurek (2009), entre otros.

6 Existen diferentes definiciones para referirse a la Región Metropolitana de Buenos Aires. Por ejemplo, la denominación AMBA incluye a la CABA y 32 partidos de la Provincia de Buenos Aires, Conurbano Bonaerense excluye a la Ciudad de Buenos Aires de los Partidos del Gran Buenos Aires o según el INDEC, el conjunto Aglomeración Gran Buenos Aires contiene además de la Ciudad de Buenos Aires 24 partidos del Gran Buenos Aires.

7 A grandes rasgos podemos identificar grandes machas urbanas con asentamientos precarios (villas, barrios populares, etc.) colindando con conjuntos habitacionales cerrados (countries, barrios cerrados). 
8 Según datos del Censo Nacional de Población y Vivienda (2010): 67\% de las viviendas estaba conectada a la red pública de agua, $38 \%$ con desagües cloacales y un $61 \%$ con gas natural.

9 Las organizaciones sociales y los gobiernos locales se configuran como ámbitos de ordenamiento para el acceso a recursos, servicios y participación en el espacio público.

10 El proyecto se enmarcó en una convocatoria para el fortalecimiento de acciones de vinculación y de desarrollo tecnológico y social de la Universidad Nacional de General Sarmiento.

11 Este proyecto fue seleccionado y financiado por el Programa de Articulación y Fortalecimiento Federal de las Capacidades en Ciencia y Tecnología COVID-19 del Ministerio de Ciencia, Tecnología e Innovación de la Nación.

12 Se desarrollaron cursos de capacitación para referentes del barrio, comedores, ollas populares, organizaciones sociales que combinaban encuentros sincrónicos con diferentes actividades de lectura y reflexión. Estos cursos se realizaron a través de grupos cerrados en la red social Facebook.

13 Entre las diferentes actividades del proyecto se diseñó un formulario para registrar a los y las participantes del curso de capacitación; se organizó la entrega a las organizaciones sociales de una caja con diferentes elementos: tapabocas con la marca del proyecto, barbijos quirúrgicos, alcohol en gel y una carpeta con materiales de capacitación; el diseño de una campaña "Tenemos un Plan" para situar la información preventiva en los escenarios reales de la vida cotidiana; entre otras.

14 Sumadas a Potenciar Trabajo; Programa de Mejoramiento de Barrios; Ingreso Familiar de Emergencia; Programa Argentina Unida por la Integración de Barrios Populares; entre otras. 